\title{
Remote Control and Automatic Monitoring of Earth-moving Machines in Road Construction
}

\author{
Marco Gatti(*), Fiorenzo Malaguti(**) \\ *Department of Engineering, Ferrara University, via Saragat 1, 44100 Ferrara, Italy \\ Email: mgatti@ing.unife.it \\ **CNR - IMAMOTER, via Canal Bianco 28, 44044 Cassana (Ferrara), Italy \\ Email:F.Malaguti@imamoter.cnr.it
}

\begin{abstract}
We report a system for remote control and automatic management of a fleet of earth-moving and construction machines with assisted guidance in earthworks and road construction. The system can be divided into three segments: control segment, positioning segment, data transmission segment. The positioning segment is based on WAAS or classical DGPS techniques. The transmission segment consists of GSM and fixed telephone network modems. The 3D positions of the machines, calculated in real time, are sensed at the control segment, at the same time as the differential corrections, by a single device built for this application. The control segment is based on a computer and a skilled operator running GIS-CAD software which controls the working accuracy of the earth-moving or operating processes with respect to the planned profile of the works. Laboratory and field tests were carried out to simulate the working performance of the system: during the tests, the 3D positions were considered with differential corrections from the WAAS/EGNOS system and with DGPS RTCM differential corrections via Internet. In this paper, we describe the system architecture and the preliminary experimental results.
\end{abstract}

KEY WORDS: Earth-moving Machines; GPS; Internet; Remote Control; Total Station; WAAS/EGNOS.

\section{INTRODUCTION}

There are many systems for the management of vehicle fleets and they are usually designed for specific applications. For example, Ashtech proposes a system for commercial fleets based on differential GPS positioning [Bouquet]: transmission of the positions occurs by UHF modems. For construction machinery, Caterpillar [Caterpillar] [Greene 2001], DSNP [Hintzy 1999a] and Leica Geosystem propose solutions based on accurate real-time positioning by GPS or laser systems. In the latter examples the GPS technique is used in a direct RTK way [Greene 1998] or an inverted one [Bradley]. It is well known that this technique requires a fixed receiver connected with mobile receivers on the machines, with the exception of a system based on one-way RTK [Sharpe]. The laser systems [Peck 1995] consist of an automated total station (theodolite) placed on a point with known co-ordinates and a prism with $360^{\circ}$ reception mirrors placed on the operating machine. During the operations the total station pursues the prism and sends its position to the control computer. The prism and theodolite must be in constant visual contact; in the presence of fixed obstacles, the theodolite is able to track the prism only when it again becomes visible.
The nominal accuracy of the two systems, usually centimetric in the plane and in height [Peck 1997] [Hintzy 1999-b], is assured inside a radius of 10 $\mathrm{km}$ for GPS and about 300 meters for laser systems. Unlike laser positioning, the GPS can work in all operating conditions if the receiver is able to record the signals of a sufficient number of satellites. The decreasing cost of GPS receivers, the development of differential positioning techniques based on geostationary satellites at free rent or via Internet, and the progress in wireless communication have allowed the creation of lowcost systems for the management and control of vehicle fleets. The aim of this work is to describe one of these systems, designed and built at the Engineering Department of Ferrara University, for real-time remote control of the $3 \mathrm{D}$ positioning of one or more construction machines, e.g. earthmoving machines used to fill road beds. The first part of the paper describes the system components while the second part reports the functional tests carried out with an agricultural tractor. The tests consider two type of DGPS positioning, the first based on use of the European WAAS system, named EGNOS, and the second based on differential corrections via Internet. 


\section{SYSTEM ARCHITECTURE}

The system is composed of three segments: Control, Positioning and Data Transmission.

\subsection{Control Segment}

The control segment receives the positional data from the operating machines and transmits the messages of DGPS corrections. Its components are:

- a PC with Windows operating system;

- the commercial software Arcview 3.2 with ArcTracking extension and VisualGPS 3.33.

Both programs acquire NMEA coded data of GPS receivers via a serial port and display the 3D positions of the receivers in real time on a georeference map. The software can manage many vehicles and it is also possible to record their paths to display them later on;

- an Internet/Intranet 10/100 Mb 3Com Board;

- some more or less detailed numerical maps of the test area, conveniently georeferenced.

The information is received by a modem via fixed or mobile telephony, or by Internet/Intranet. During the tests, the control segment was placed inside a building close to the test area. Figure 1 shows a photo of the control segment.

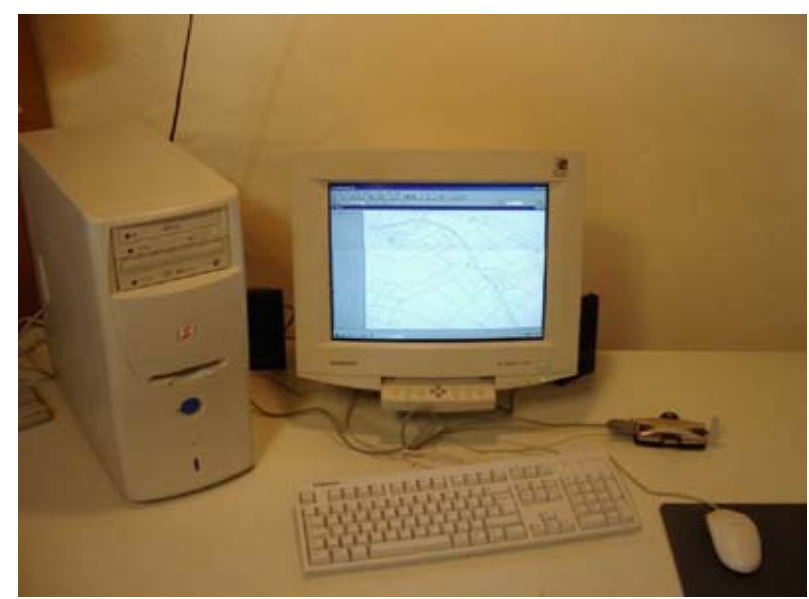

Figure 1. Control segment

\subsection{Positioning Segment}

The positioning segment provides real-time data on the position and velocity of the fleet. The data are transmitted to the control segment, which uses them to organise and plan the work tasks of the construction machines.

For real-time positioning of the machines, two solutions were considered: a) automated theodolite;

b) differential GPS.

In the end we chose the differential technique in the WAAS and classic GPS modes; the latter uses RTCM corrections received via Internet. The GPS technique seemed the most suitable to satisfy the operational and accuracy requirements of construction works, usually characterised by low and medium accuracy.

The parts of the positioning segment are:

- a low-cost receiver, model Etmac Crux-2, built at the Engineering Dept. of Ferrara University [Gatti]. It is an L1 monofrequency receiver, with $\mathrm{C} / \mathrm{A}$ code and 12 channels. The receiver can work in classical Differential GPS mode and Wide Area DGPS, with differential corrections from WAAS/EGNOS. In both cases the accuracy of 3D positioning is a few meters. The receiver is powered by external lead batteries, which assure 8 hours of work; in the near future it will be powered by the battery of the vehicle. The data transmission of positions can be in proprietary or NMEA formats;

- an Ashtech kinematic antenna, Marine model, part number 700700c;

- a Psion Epoc5 palmtop, with 16 Mb RAM, 37 $\mathrm{MHz}$ processor and monochromatic LCD screen. The palmtop, connected to the receiver by the RS232 serial port, functions as an external controller, proving to be less fragile and more handy than a portable PC;

- the palmtop runs RealMaps navigation software, which allows one to view the vehicle's position on a map directly on the Psion's screen. Thus the Psion and RealMaps function as a remote control unit, partly replacing the control segment.

Figure 2 shows a photo of the positioning segment.

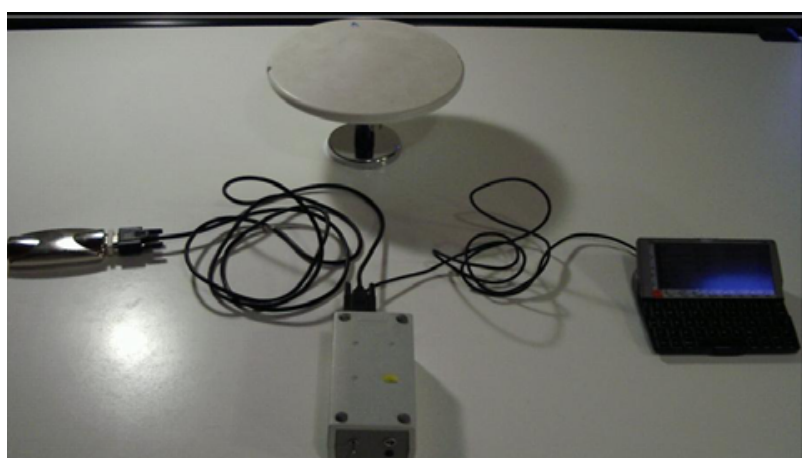

Figure 2. Positioning segment 


\subsection{Transmission Segment}

The transmission segment allows transmission of the positional and velocity data remotely from the machine to the control segment, and vice versa the RTCM corrections from the control segment to the machine. In general the transmission can occur via UHF radio modem, via a modem using fixed or mobile telephone networks, via geostationary telecommunication satellites or via Internet. In the absence of large obstacles, UHF radio modems allow signal reception within $10-15 \mathrm{~km}$ from the transmitting station. The disadvantages of radio modems are the need to install and service radio repeaters, to obtain government licences, to increase the complexity of the system with natural obstacles; the advantages are that they work even with natural disasters, which could overload and/or damage the other communication networks, the low transmission costs, the ability also to transmit digital or voice messages from/to the vehicles.

Data transmission via geostationary satellites needs special devices, with higher transmission costs than mobile communications. Yet it has the great advantage of covering the entire terrestrial surface.

Data transmission and reception via Internet is the most recent application [Hada]. With "Server" software it is possible to send data to a remote station using "Client" software. Both the receiving and transmitting stations must be connected via Internet.

To set up and develop the transmission segment we chose the solution based on fixed and mobile telephone networks. Two types of modem were used:

- a modem for fixed telephony, US Robotics 56k by $3 \mathrm{COM}$, connected to a PC via RS232 serial port and software for serial communication. The modem adapts its transmission rate to the line conditions;

- a modem for mobile telephony, Falcom Twist, connected to the GSM network via SIM card for data transfer. The maximum transfer rate is 9600 bps, but the modem can work in GPRS mode with a higher transmission rate. However $9600 \mathrm{bps}$ is sufficient for the transfer of differential corrections and NMEA data. The GSM modem has a serial port controlled by a serial communication program. The modem can also be powered by a $12 \mathrm{~V}$ lead battery.

Figure 3 shows a photo of the transmission segment.
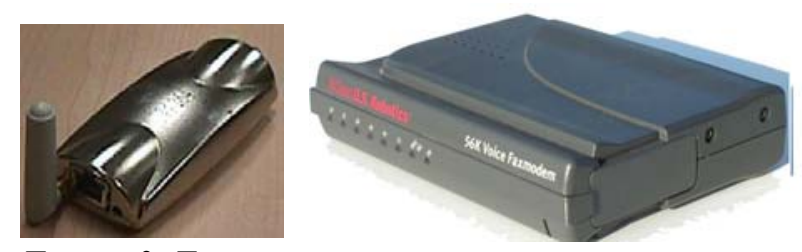

Figure 3. Transmission segment

Finally, Figure 4 is a block diagram of the entire system.

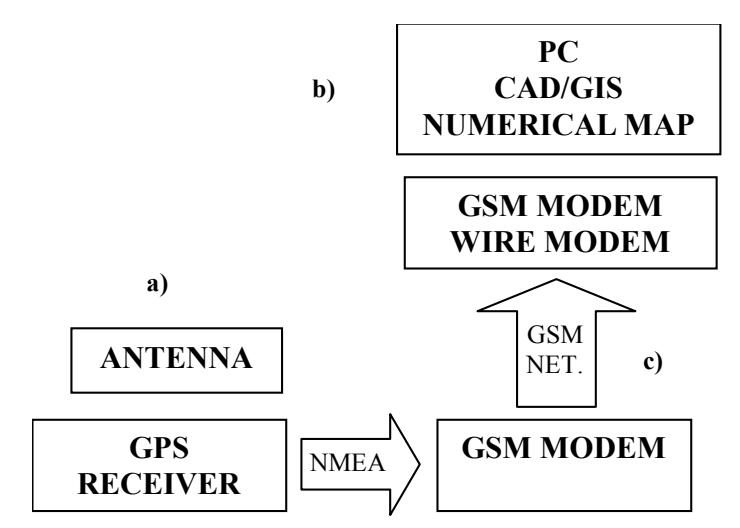

Figure 4. Block diagram of the entire system.

\section{EXPERIMENTAL TESTS}

\subsection{Description of the test}

Many laboratory and field tests were conducted to simulate the working performance of the system. The field tests were carried out at a farm near Ferrara using a New Holland TN75D agricultural tractor as a four-wheel "rover" vehicle. The test paths were chosen inside the farm.

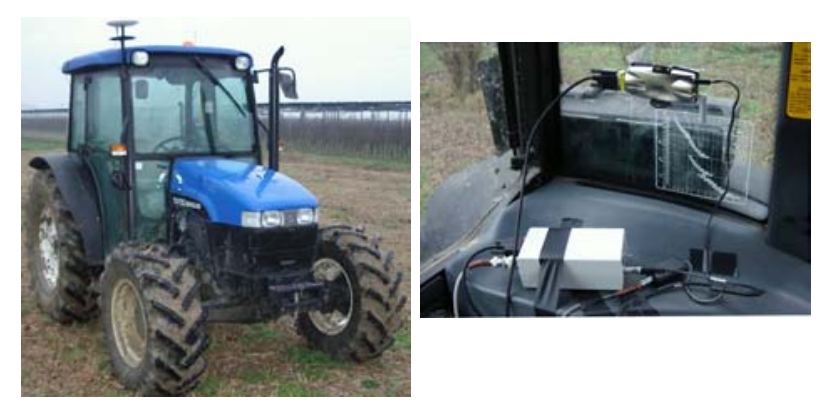

Figure 5. Tractor equipped with the kinematic antenna. Detail of the GSM modem and EMTAC receiver inside the driver's cabin.

The kinematic antenna was fixed to the top of the tractor cabin by a magnetic support (Fig. 5). The EMTAC receiver, Falcom Twist modem (to transmit the vehicle positions) and their lead batteries were placed inside the cabin. The control 
station was set up in a nearby farm building and connected via a second Falcom Twist modem.

In the first test the EMTAC receiver was set in WADGPS mode with reception of differential corrections from the geostationary satellite EGNOS AOR EST PRN 120. Figure 6 is a block diagram of the components used in the first test.

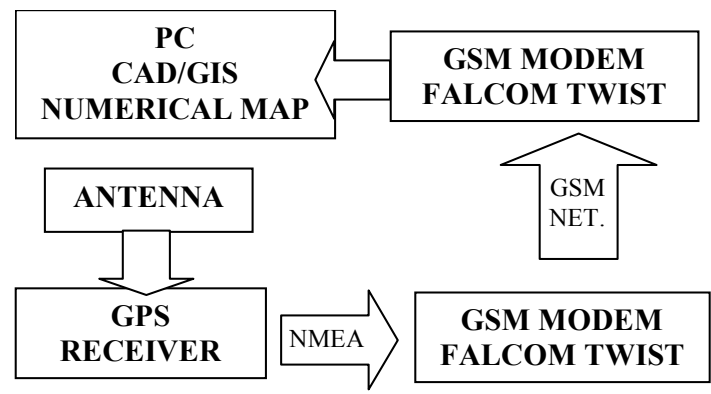

Figure 6. Block diagram of the components used in the first test.

In the second test the receiver was set in DGPS mode, with reception of the differential corrections from the permanent GPS station of Turin via Internet [Rupprecht][Lee]. A second modem was placed in the tractor to receive the DGPS-RTCM differential corrections and direct them to another serial port of the GPS receiver (obviously different from the port outputting the positions). For the second test it was necessary to set up a second fixed station at the Engineering Department to receive the differential corrections from the Turin station via Internet and forward them to the "rover" vehicle via a US Robotics Fax Modem $56 \mathrm{~K}$ and the fixed telephone network. The control station used a US Robotics 56K Voice Fax modem to connect to the fixed telephone network. The block diagram of the components used for the second test is shown in Figure 7.

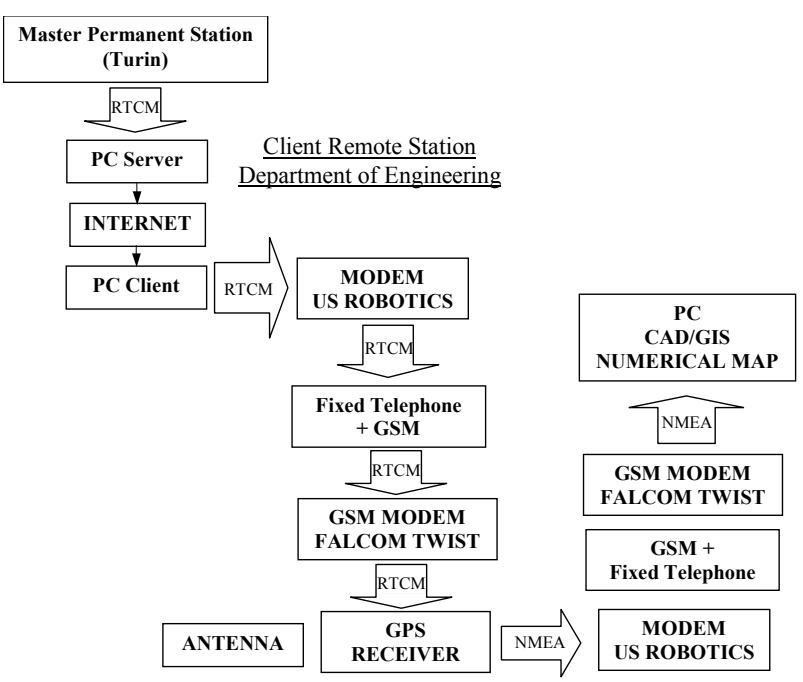

Figure 7. Block diagram of the components used in the second test.

It can be seen that the station at the Engineering Dept. sends the RTCM transmission to the first GSM modem in the vehicle, while the second GSM modem transmits the DGPS positions to the control station where they are displayed on the map by ArcView. In both tests, NMEA messages were transmitted using GGA and GSA protocols, with a sampling rate of 1 second.

\subsection{Test results}

The results of the two tests were first evaluated as the percentage of positions sent to the control segment and the latency of the position transmissions, which means a delayed response of the control segment. Table 1 shows the results of both tests.

Table 1. Results of the two tests.

\begin{tabular}{|c|c|c|c|}
\hline \multicolumn{2}{|c|}{ Test } & I & II \\
\hline \multicolumn{2}{|c|}{ Period [seconds] } & $\mathbf{1 6 8 6}$ & $\mathbf{7 5 7}$ \\
\hline \multicolumn{2}{|c|}{ Position (\%) } & $\mathbf{1 0 0}$ & $\mathbf{1 0 0}$ \\
\hline \multicolumn{2}{|c|}{ Path length } & $\mathbf{3 , 2} \mathrm{km}$ & $\mathbf{2 , 6} \mathbf{~ k m}$ \\
\hline Transmission & min & $\mathbf{1 , 0}$ & $\mathbf{1 , 1}$ \\
\cline { 2 - 4 } latency & medium & $\mathbf{1 , 5}$ & $\mathbf{2 , 6}$ \\
\cline { 2 - 4 } [seconds] & $\mathbf{m a x}$ & $\mathbf{2 , 5}$ & $\mathbf{3 , 5}$ \\
\hline
\end{tabular}

A comparison was then made to assess the horizontal and vertical accuracy. Figure 8 shows part of the path covered by the machine during the two tests. 


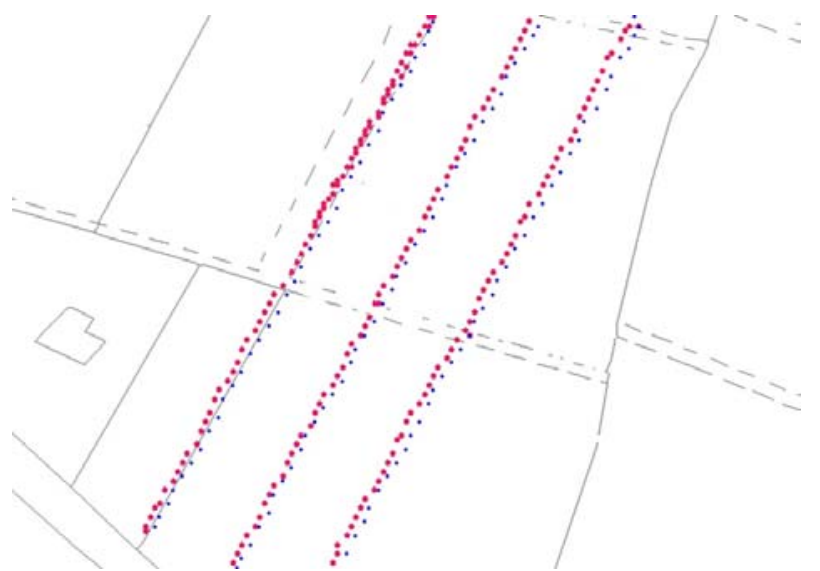

Figure 8. Comparison between the positions obtained with WAAS/EGNOS corrections (red dots) and DGGPS-RTCM positions (blue dots).

The map used is a vector cadastral map georeferenced in the WGS 84 system The results for height are shown in Figure 9.

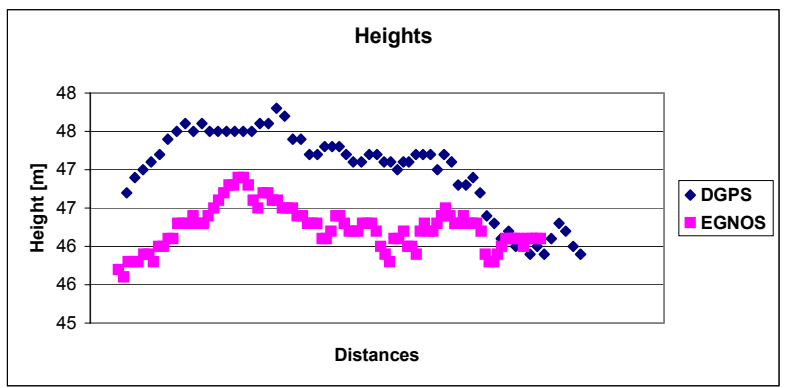

Figure 9. WADGPS and DGPS heights.

\section{CONCLUSION}

The positioning by EGNOS differential corrections is certainly simpler to obtain, using less resources and means; one only needs a GSM connection and two modems (Figure 6). However the geostationary satellites could be blocked by obstacles.

The positioning by DGPS via Internet requires two GSM connections and thus greater means and costs. Moreover the latency increases and consequently the response time of the control segment is delayed. It is possible to acquire receivers with a bidirectional port, so that the differential corrections and NMEA positions can be received and transmitted by the same communication port. This would allow a single connection for the data transfer, but these receivers are rather expensive.
Regarding the horizontal and vertical accuracy, the DGPS positioning via Internet produces a more uniform or less spread recording than the EGNOS positioning (Figure 8). The comparison of the heights, made on the same $250 \mathrm{~m}$ path (Figure 9), shows an initial variation of the DGPS positioning, but after that the height differences between the two tests are nearly constant.

\section{ACKNOWLEDGEMENT}

Thanks to Dr. Andrea Chiorboli for the execution and description of the field tests.

\section{REFERENCES}

[Bradley] Bradley, W.J., Smith, K.H., Tiware, A., 1999, Wide Area Inverse DGPS for Fleet Operations. TechnoCom Corporation, USA.

[Bouquet] Bouquet, P., 1999, Le GPS Différentiel au Coeur des Noveaux Systèmes de Gestion de Flotte Terrestre. Revue Navigation, Vol.47, n.186, april 1999.

[Carlson] Carlson, B., Baertlein, H., Eckels, R., Lyle, S., 1999, Dozer 2000 - A Revolution in Machine Guidance. Leica Geosystem, 1999.

[Caterpillar] Caterpillar Inc., 2000, Caterpillar's Computer Aided Earthmoving System for Landfills. Concord CA (USA), www.cat.com.

[Gatti] Gatti, M., Tracchi, L., 2003, A Low-Cost GPS Receiver for Remote Control of EarthMoving Machines. In press to Italian Institute of Navigation Review.

[Greene 1998] Greene, D., 1998, Benefits of GPS Position Monitoring for Earthmoving Equipment. Bulletin of Society for Mining, Metallurgy and Exploration Inc., March 1998, Littleton CO (USA).

[Greene 2001] Green, D., 2001, Value of GPS and Computer Aided Earthmoving Systems in Landfills. SWANA IV Annual Landfill Symposium, 18 June 2001.

[Hintzy 1999-a] Hintzy, G., Pichot, C., 1999-a, Guidage d'engins de travaux publics par GPS: l'exploitation optimale des parcs de machines. RGRA Revue General des Routes, n.771 (France), March 1999. 
[Hada] Hada, H. et al., 1999, Differential and RTK for the Internet Car. Proceedings of GNSS 1999, Vol.2, October 1999, Genoa.

[Hintzy 1999-b] Hintzy, G., Noury, J.M., Pichot, C., 1999-b, Improving Work Conditions, Productivity and Quality at Construction Sites. Remote Machine Guidance by Real-Time Kinematic GPS. Gim International, March 1999;

[Lee] Lee, Y, et al., 2000, Internet based DGPS for mobile communication user. Proceedings of ION GPS 2000, Salt Lake City, UT, 19-22 September 2000.

[Peck 1995] Peck, J., Gray, J., 1995, The Total Mining System (TMS): the base for open pit automation. CIM Bulletin, Vol.88, Num.993, pp.38-44 Montreal, Quebec (Canada).

[Peck 1997] Peck, J., Hendricks, C., 1997 Applications of GPS-based Navigation Systems on mobile mining equipment in open-pit mines. CIM Bulletin Vol.90 Num.1011 pp.114-119, June 1997 Montreal, Quebec (Canada).

[Rupprecht] Rupprecht, W., 1999, DGPS corrections over the Internet. http://www.wsrcc.com/wolfgang/gps/dgps-ip.html.

[Sharpe] Sharpe, T., Hatch, R., Nelson, F., 2000, John Deere's StarFire System: WADGPS for Precision Agriculture. $13^{\text {th }}$ International Meeting of the Satellite Division of the ION Institute of Navigation, 19-22 September 2000, Salt Lake City, USA. 